\title{
Paeonol promotes microRNA-126 expression to inhibit monocyte adhesion to ox-LDL-injured vascular endothelial cells and block the activation of the PI3K/Akt/NF-kB pathway
}

\author{
XUSHENG YUAN ${ }^{1,2}$, JUNJUN CHEN ${ }^{2}$ and MIN DAI ${ }^{1,2}$ \\ ${ }^{1}$ Key Laboratory of Chinese Medicine Research and Development, and ${ }^{2}$ Key Laboratory of Xin'an Medicine, \\ Department of Pharmacy, Anhui University of Chinese Medicine, Hefei, Anhui 230038, P.R. China
}

Received September 10, 2015; Accepted October 3, 2016

DOI: $10.3892 / \mathrm{ijmm} .2016 .2778$

\begin{abstract}
Paeonol (2'-hydroxy-4'-methoxyacetophenone) is an active component isolated from the root of Paeonia Suffruticosa Andrews. We previously found that paeonol inhibited vascular cell adhesion molecule-1 (VCAM-1) expression, and thus may be useful for the prevention and treatment of rabbit atherosclerosis (AS); however, the underlying mechanisms are not yet well known. Recently, microRNAs (miRNAs or miRs) have been reported to play an important role in the pathogenesis of AS. In the present study, we examined the effects of paeonol on miRNA126 (miR-126) expression, and its ability to inhibit monocyte adhesion to oxidized low-density lipoprotein (ox-LDL)-injured vascular endothelial cells (VECs). VECs were isolated from the rat thoracic aorta and stimulated with ox-LDL ( $20 \mathrm{mg} / \mathrm{l})$ in the presence of paeonol. We found that miR-126 had a lower expression in the ox-LDL-injured VECs, and VCAM-1 was identified as a target gene of miR-126. Furthermore, paeonol promoted miR-126 expression and suppressed VCAM-1 expression at the mRNA and protein level. It also inhibited monocyte adhesion to ox-LDL-injured VECs through the promotion of miR-126 expres-
\end{abstract}

Correspondence to: Professor Min Dai, Key Laboratory of Chinese Medicine Research and Development, Department of Pharmacy, Anhui University of Chinese Medicine, 103 Meishan Road, Hefei, Anhui 230038, P.R. China

E-mail: daiminliao@163.com

Abbreviations: Akt, protein kinase B; ox-LDL, oxidized lowdensity lipoprotein; FBS, fetal bovine serum; AS, atherosclerosis; DMEM, Dulbecco's modified Eagle's medium; DMSO, dimethylsulfoxide; I $\mathrm{B} \alpha$, inhibitory factor $\kappa \mathrm{B}-\alpha$; miRNAs or miRs, microRNAs; IL, interleukin; MTT, 3-(4,5-dimethyl-2-thiazolyl)-2,5diphenyl-2-H-tetrazolium bromide; NF- $\kappa \mathrm{B}$, nuclear factor- $\kappa \mathrm{B}$; PDTC, pyrrolidinedithiocarbamate; PI3K, phosphatidylinositol 3-kinase; TNF- $\alpha$, tumor necrosis factor- $\alpha$; VSMCs, vascular smooth muscle cells; VCAM-1, vascular cell adhesion molecule-1; VECs, vascular endothelial cells

Key words: paeonol, vascular endothelial cells, vascular cell adhesion molecule-1, microRNA-126, phosphatidylinositol 3-kinase/protein kinase $\mathrm{B} /$ nuclear factor- $\kappa \mathrm{B}$ pathway sion. Furthermore, it was demonstrated that paeonol blocked the activation of the phosphatidylinositol 3-kinase (PI3K)/protein kinase $\mathrm{B}(\mathrm{Akt}) / \mathrm{nuclear}$ factor- $\mathrm{\kappa B}(\mathrm{NF}-\mathrm{\kappa B})$ signaling pathway by promoting miR-126 expression. Taken together, and to the best of our knowledge, the findings of this study provide the first evidence that paeonol promotes miR-126 expression to inhibit monocyte adhesion to ox-LDL-injured VECs and block the activation of the PI3K/Akt/NF- $\mathrm{kB}$ signaling pathway. Our data suggest that miR-126 plays a crucial role in vascular inflammation and may be an important therapeutic target in the treatment of AS with the use of paeonol.

\section{Introduction}

Paeonol (2'-hydroxy-4'-methoxyacetophenone) (chemical structure shown in Fig. 1) is a main constituent of the root of Paeonia Suffruticosa Andrews, which has long been used as an anti-inflammatory agent in China $(1,2)$. We previously found that paeonol was effective in the prevention and treatment of atherosclerosis (AS) at the initiation and developmental stages through the inhibition of the inflammatory response in animal models. Paeonol inhibited vascular cell adhesion molecule-1 (VCAM-1) expression to suppress monocyte adhesion to tumor necrosis factor- $\alpha$ (TNF- $\alpha$ )-stimulated vascular endothelial cells (VECs) (3). However, the underlying mechanisms are not yet well known. It has been demonstrated that paeonol can suppress serum and aorta lipid peroxidation in models of hyperlipidemia, and can decrease serum oxidative modification induced by oxidized low-density lipoprotein (ox-LDL) in healthy humans (4-6). Paeonol has also been shown to inhibit the expression of interleukin (IL)- $1 \beta$ and TNF- $\alpha$ to inhibit the over-proliferation of vascular smooth muscle cells (VSMCs), and decrease local inflammation in AS (7).

VCAM-1 belongs to the immunoglobulin superfamily and is only expressed in VECs (8), and it can be stimulated by cytokines, thus playing an important role in the pathogenesis of AS $(9,10)$. MicroRNAs (miRNAs or miRs) are small non-coding RNAs that regulate the post-transcription of target genes $(11,12)$, and have been shown to be involved in the pathogenesis of AS and to regulate the biological functions of VECs (13). miRNA-126 (miR-126) is located in an intron of the epidermal growth factor like domain-7, and is selectively 
expressed in VECs (14). The knockdown of miR-126 has been shown to induce the proliferation and migration of VECs, which results in endangium damage and angiogenesis-dependent disorders (15). Notably, VCAM-1 is a specific target gene of miR-126, and the inhibition of miR-126 expression leads to a higher expression of VCAM-1 in TNF- $\alpha$-stimulated VECs (11). Moreover, phosphatidylinositol 3-kinase (PI3K) is one of the most relevant players in VEC biological processes, and activates protein kinase B (Akt) (16). The transcription factor, nuclear factor- $\mathrm{\kappa B}(\mathrm{NF}-\kappa \mathrm{B})$, participates in cellular processes, such as cell adhesion (17).

However, it has been reported that the expression of miRNAs can be regulated by natural compound (18). In this study, we examined the effect of paeonol on miR-126 expression, and its ability to inhibit monocyte adhesion to ox-LDL-injured VECs. Thus far, the underlying mechanisms responsible for the regulatory effects of paeonol on miR-126 expression have not yet been fully elucidated. Ox-LDL was used for the stimulation of VECs, as its formation in the subendothelial space of vascular wall is a key stage in the pathogenesis of AS (19). Our findings indicate that miR-126 may be an important target in treatment of AS with the use of paeonol.

\section{Materials and methods}

Animals. At 6-8 weeks after birth, a total of 20 male Sprague-Dawley rats (weighing 160-180 g) were obtained from the Experimental Animal Center of Anhui Medical University (Hefei, China). All rats were maintained under specific-free conditions in the animal facility at the Anhui University of Chinese Medicine. They were housed in a polypropylene cage $\left(25 \pm 5^{\circ} \mathrm{C}, 40-50 \%\right.$ relative humidity) under controlled lighting (12 h light/dark cycle), and allowed free access to food and water. All animal experiments were carried out in accordance with the Guide for the Care and Use of Laboratory Animals prepared by the National Academy of Sciences and were approved by the Ethics Committee of Anhui University of Chinese Medicine (Hefei, China).

Chemicals. Paeonol (purity, 99\%) was obtained from Baicao Plants Co., Ltd. (Xuancheng, China). Dulbecco's modified Eagle's medium (DMEM) was purchased from Gibco Life Technologies Co. (Grand Island, NY, USA). ox-LDL (YB-002-1) waspurchasedfrom YiyuanBiotechnologiesCo.,Ltd.(Guangzhou, China). HiPerFect transfection reagent, miScript miR-126 mimic and inhibitor, the miRNeasy mini kit (Cat. no. 217004) and the miScript PCR Starter kit (Cat. no. 218193) were purchased from Qiagen Co., Ltd. (Dusseldorf, Germany). The Dual-Luciferase Reporter kit was purchased from Promega Biotech Co., Ltd. (Madison, WI, USA), and the constructs of wild- and mutant-type 3'UTR of VCAM-1 mRNA were obtained from Huaan Pingkang Biotechnology Co., Ltd. (Shen zhen, China). The PI3K inhibitor, LY294002, and ammonium pyrrolidinedithiocarbamate (PDTC), a selective NF- $\kappa$ B inhibitor, were purchased from Beyotime Biotech, Inc. (Shanghai, China). Antibody to p-PI3K (Cat. no. 4228L) was purchased from Cell Signaling Technology, Inc. (Danvers, MA, USA). Antibodies to NF-kB p65 (Cat. no. BS9879M), inhibitory factor $\kappa \mathrm{B}-\alpha(\mathrm{I} \kappa \mathrm{B} \alpha$; Cat. no. BS3601), p-IкB $\alpha$ (Cat. no. BS4736) and VCAM-1 (Cat. no. BS6005) were purchased from Bioworld

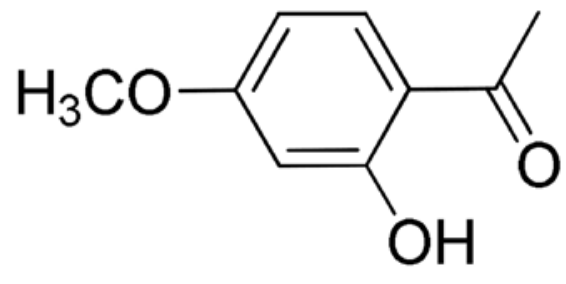

Figure 1. The chemical structure of paeonol.

Technology Co., Ltd. (Louis Park, MN, USA). Antibodies to PI3K (Cat. no. sc-48639), Akt (Cat. no. sc-24500), p-Akt (Cat. no. sc-7985-R) were purchased from Santa Cruz Biotec hnology, Inc. (Santa Cruz, CA, USA). All other reagents were purchased from reliable companies.

Isolation of rat VECs. The VECs were isolated from the rat thoracic aorta as follows: the rats were sacrificed by euthanasia. The thoracic aortas were removed from the rats using surgical scissors, and the clean blood vessels were obtained from stripped connective and fatty tissues under sterile conditions, reversed and stringed at both ends tightly. The vascular intima was washed with DMEM supplemented with $10 \%$ fetal bovine serum (FBS), and incubated to digestion at $37^{\circ} \mathrm{C}$, and $5 \% \mathrm{CO}_{2}$ for $1 \mathrm{~h}$ in $0.2 \%$ collagenase I and rinsed using medium. After cutting off, the remaining aorta was cut into sections, and the tissue fragments were incubated under the same conditions. Once the cells had formed a monolayer, the explants were removed, and the cells were subcultured. The cells were identified by their endothelial cell-specific, cobblestone-like morphology, and the cells were determined by the immunocytochemical staining for Factor VIII (Boster, Wuhan, China). The cells were collected by centrifugation and resuspended in DMEM containing 10\% FBS for the investigation. In all experiments, the VECs were used at passages 3-5.

3-(4,5-Dimethyl-2-thiazolyl)-2,5-diphenyl-2-H-tetrazolium bromide (MTT) assay. The VECs were seeded in 96-well plates at a density of $1 \times 10^{4}$ cells/well in $200 \mu 1$ DMEM overnight to allow attachment. The cells were then treated with fresh medium containing various concentrations of 5, 10,20, 40 and $80 \mathrm{mg} / \mathrm{l}$ of ox-LDL and cultured for 12,24 and $48 \mathrm{~h}$, or pre-treated with various concentrations of $15,30,60,120,240$ and $480 \mu \mathrm{M}$ of paeonol and then cultured for 12, 24 and $48 \mathrm{~h}$ as above. MTT solution was added to each well followed by incubation for $4 \mathrm{~h}$. The growth medium was then removed and replaced with dimethylsulfoxide (DMSO), followed by incubation for $10 \mathrm{~min}$. A microplate reader (SpectraMax Mze; Molecular Devices, Sunnyvale, CA, USA) was used to measure the absorbance $(A)$ at $490 \mathrm{~nm}$. Cell viability was calculated as follows: relative viability $(\%)=A_{490}($ test $)-A_{490}($ normal $) / A_{490}($ control $)-A_{490}($ normal $) \times 100$.

Transient transfection. The VECs were seeded in 6-well plates at a density of $1 \times 10^{5}$ cells/well in the presence of $2 \mathrm{ml}$ DMEM containing 10\% FBS, and incubated under normal conditions (typically $37^{\circ} \mathrm{C}$ and $5 \% \mathrm{CO}_{2}$ ). HiPerFect transfection reagent $(12 \mu \mathrm{l})$ was added to the culture medium without serum to a volume of $300 \mu 1$. Subsequently, $0.6 \mu 1$ of the miR-126 mimic (20 $\mu \mathrm{M}$ stock solution) or $6 \mu \mathrm{l}$ of the 
miR-126 inhibitor ( $20 \mu \mathrm{M}$ stock solution) were added to these mixtures, and mixed by vortexing. The complexes were added to the VEC medium and gently swirled. The VECs were then incubated with the transfection complexes for $24 \mathrm{~h}$, and these media were changed as required.

Quantitative polymerase chain reaction ( $q P C R)$. Total RNA was extracted from the VECs using the Qiazol lysis reagent (Qiagen, Dusseldorf, Germany). cDNA prepared in a transcript reaction using miScript PCR Starter kit (Qiagen) served as the template for qPCR analysis. The reverse transcription conditions were as follows: $37^{\circ} \mathrm{C}$ for $60 \mathrm{~min}$, and $95^{\circ} \mathrm{C}$ for $5 \mathrm{~min}$. Subsequently, the cDNA was used for the quantification of miR-126 and VCAM-1 mRNA expression. The amplification conditions were $95^{\circ} \mathrm{C}$ for $15 \mathrm{~min}$ at the initial activation, followed by 40 cycles of $94^{\circ} \mathrm{C}$ for $15 \mathrm{sec}, 55^{\circ} \mathrm{C}$ for $30 \mathrm{sec}$, and $70^{\circ} \mathrm{C}$ for $30 \mathrm{sec} . \beta$-actin primers were used as an internal control in equal amounts to those of the mRNA used. Gene expression was analyzed using QuantiTect SYBR-Green PCR master mix miRNA or mRNA assays, according to the manufacturer's instructions (Applied Biosystems, Foster City, CA, USA). Dissociation curve analysis of each sample yields only one peak, resulting from the specific amplification product (primer-dimer not co-amplified). These values were normalized to the reference gene $\beta$-actin as follows: fold $=2^{-\Delta \Delta \mathrm{Ct}}$, where $\Delta \mathrm{Ct}$ represents the differences in cycle threshold numbers between each of the target gene and $\beta$-actin, and $\Delta \Delta \mathrm{Ct}$ represents the relative change between compared groups.

Western blot analysis. The VECs were lysed in ice-cold RIPA buffer (1\% NP-40, $0.1 \%$ sodium dodecyl sulfate, $0.5 \%$ sodium deoxycholate and $50 \mathrm{mM}$ Tris, $\mathrm{pH}$ 7.4) containing protease inhibitor phenylmethanesulfonyl fluoride. The total protein was quantified by BCA assay, and the loaded concentration was $300-500 \mu \mathrm{g} / \mathrm{ml}$. The protein was separated by $10 \%$ sodium dodecyl sulfate-polyacrylamide gel electrophoresis. The protein was transferred onto a polyacrylamide difluoride membrane, which was blocked in phosphate-buffered saline-Tween-20 containing 5\% non-fat dry milk, and incubated with primary antibodies to VCAM-1, p-PI3K, PI3K, p-Akt, Akt, p65, $\mathrm{p}-\mathrm{I} \kappa \mathrm{B}, \mathrm{I} \kappa \mathrm{B}$ or $\beta$-actin. Immunoreactive protein was detected with secondary antibodies (Cat. nos. ZB2301 and ZB2305; Zhongshan, Beijing, China) conjugated to horseradish peroxidase which enhanced chemiluminescence. To investigate the relation between PI3K/Akt and NF- $\kappa \mathrm{B}$ pathways in the effect of paeonol, the VECs were pretreated with LY294002 (an inhibitor of PI3K, $50 \mu \mathrm{M}$ ) and PDTC (an inhibitor of NF- $\kappa \mathrm{B}, 50 \mu \mathrm{M}$ ) for $1 \mathrm{~h}$, and then treated with ox-LDL (20 mg/l) in the presence of paeonol $(120 \mu \mathrm{M})$. The protein expression levels were detected.

Generation of luciferase reporter construct. miR-126 consensus response element, UGAGUGCCAUGCU, VCAM-1 wild-type 3'UTR response element, ACUGGACGGUACGG, and VCAM-1 mutant-type 3'UTR response element, and ACU GUUGCCAUGCG, were inserted into the multiple cloning site of the miR-126 expression vector, p-LUC-Report, downstream of cDNA for luciferase. Each vector, along with miR-126 mimic, was transfected into the VECs using HiPerFect reagent. The VECs were cultured for $24 \mathrm{~h}$ and assayed using the Dual-Luciferase Reporter assay system. Relative luciferase activity was calculated by normalizing the firefly luminescence as to Renilla luminescence.

Isolation of rat monocytes. The rats were anaesthetized with chloral hydrate. The abdominal aorta blood of the rats was obtained using the heparin anticoagulant tube from the rats and centrifuged at $160 \mathrm{x} \mathrm{g}$ for $10 \mathrm{~min}$. The monocyte fraction was prepared by the Ficoll-Hypaque gradient technique. The cells were then suspended with lymphocyte separation solution (9\% sodium diatrizoate, 5.7\% Ficoll PM100), and centrifuged at $670 \mathrm{x}$ g for $15 \mathrm{~min}$. Monocytes were washed with phosphate buffer solution and DMEM, and centrifuged at $240 \mathrm{xg}$ for $6 \mathrm{~min}$. The proportion of monocytes $>90 \%$ was confirmed by flow cytometry assay using anti-CD14 (Cat. no. MAB1219) and anti-CD56 (Cat. no. MAB2120Z) antibodies (Bioworld, Nanjing, China). Cell viability $>95 \%$ was examined using trypan blue staining assay. For the following experiments, the monocyte concentration was adjusted to $1 \times 10^{6}$ cells $/ \mathrm{ml}$.

Cell adhesion analysis. The VECs were seeded in 96-well plates at a density of $1 \times 10^{4}$ cells/well, and transfected with miR-126 mimic or inhibitor. The VECs were pre-treated with paeonol for $24 \mathrm{~h}$, and incubated with fresh medium containing ox-LDL (20 mg/l) for $24 \mathrm{~h}$. The monocyte mixture was washed and co-cultured with the VECs for $60 \mathrm{~min}$ at $37^{\circ} \mathrm{C}$ and $5 \% \mathrm{CO}_{2}$. Subsequently, $200 \mu \mathrm{l}$ of PBS were added to the dishes and gently flicked on tissue paper. The unbound monocytes were removed. The adherent cells were fixed in $100 \mu \mathrm{l}$ methanol for $10 \mathrm{~min}$, washed and incubated with $100 \mu \mathrm{l}$ Rose-Bengal $(0.25 \%$, $\mathrm{pH}$ 7.3; Aladdin, Shanghai, China) for $10 \mathrm{~min}$, as previously described (20). These cells were washed 3 times with PBS and incubated with $50 \%$ ethanol for $1 \mathrm{~h}$. The absorbance $(A)$ value was measured using a microplate reader at $490 \mathrm{~nm}$.

Statistical analysis. All data are expressed as the means \pm SD, and analyzed using the SPSS version 17.0 software package. The Student's t-test and ANOVA were used for the analysis of experimental data. A value of $\mathrm{P}<0.05$ was considered to indicate a statistically significant difference.

\section{Results}

Effect of ox-LDL on miR-126 expression in VECs. To verify whether miR-126 is specifically expressed in inflammatory VECs, ox-LDL was used to stimulate the rat VECs in vitro. We found that stimulation with ox-LDL (20, 40 and $80 \mathrm{mg} / \mathrm{l})$ suppressed VEC viability in a concentration- and time-dependent manner (as shown by the increased VEC suppression rate; Fig. 2A). The suppression rate of cell viability was close to $50 \%$ in the cells stimulated with $20 \mathrm{mg} / \mathrm{l}$ ox-LDL for $24 \mathrm{~h}$. These conditions were used as the optimal stimulation concentration and time in subsequent experiments. Of note, miR-126 had a lower expression in the ox-LDL-stimulated VECs. Transfection with the miR-126 mimic significantly promoted miR-126 expression, and transfection with the miR-126 inhibitor decreased miR-126 expression (Fig. 2B). To investigate the function of miR-126, the expression of VCAM-1, which is a target gene of miR-126, was analyzed. miR-126 negatively regulated VCAM-1 expression at the mRNA and protein level (Fig. 2C and D). The expression of VCAM-1 increased with ox-LDL stimulation, and decreased 


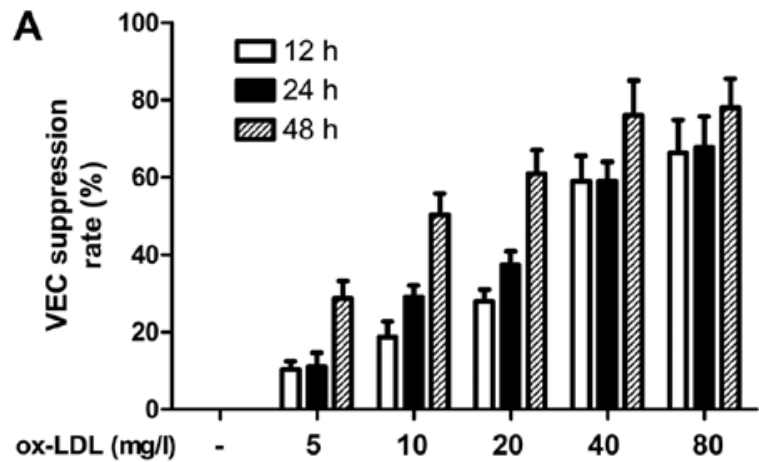

C

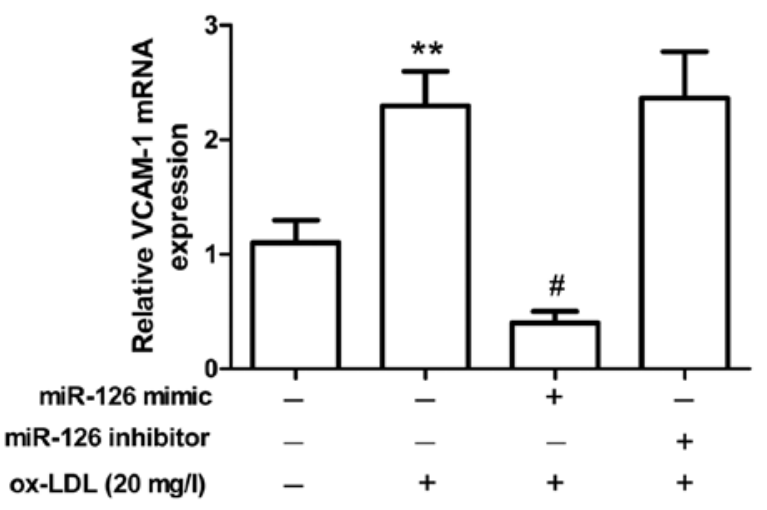

B

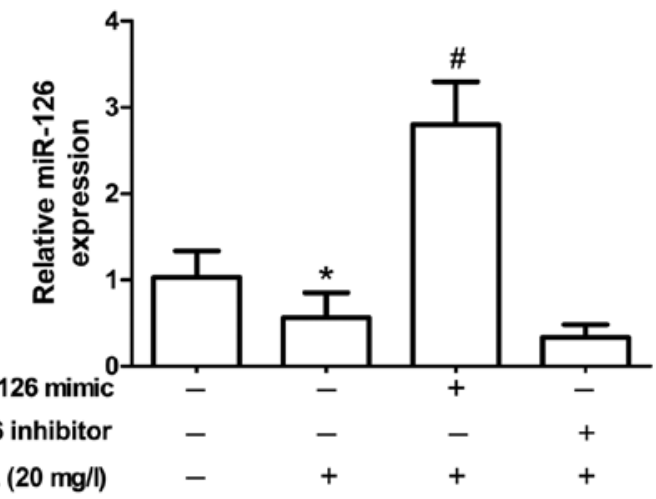

D

VCAM-1

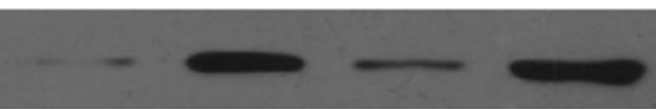

$\beta$-actin
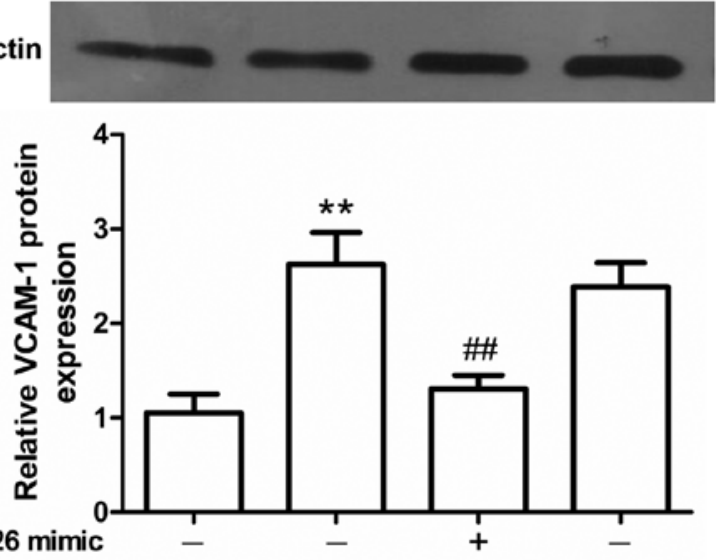

miR-126 mimic

miR-126 inhibitor

ox-LDL (20 mg/l)

Figure 2. Effect of oxidized low-density lipoprotein (ox-LDL) on miR-126 expression in vascular endothelial cells (VECs) in vitro. (A) ox-LDL (5, 10, 20, 40 and $80 \mathrm{mg} / \mathrm{l}$ ) induced obvious damage to rat VECs at 12,24 and $48 \mathrm{~h}$, as determined by MTT assay, and as shown by the increase in the VEC suppression rate. The mimic/inhibitor of miR-126 was transfected into the VECs and the VECs were then stimulated with ox-LDL (20 mg/l) for $24 \mathrm{~h}$. (B) Total RNA was harvested from the cultured VECs and the relative miR-126 expression was analyzed by qPCR. (C) Effect of miR-126 on vascular cell adhesion molecule-1 (VCAM-1) mRNA expression was examined by qPCR. (D) Total protein was harvested from cultured VECs and examined by western blot analysis for VCAM-1 protein expression, and normalized to $\beta$-actin expression. Data are expressed as the means \pm SD of at least independently triplicate experiments. "P<0.05 and ${ }^{* *} \mathrm{P}<0.01$ vs. control group (no treatment); ${ }^{*} \mathrm{P}<0.05$ and ${ }^{\# \#} \mathrm{P}<0.01$ vs. ox-LDL group.

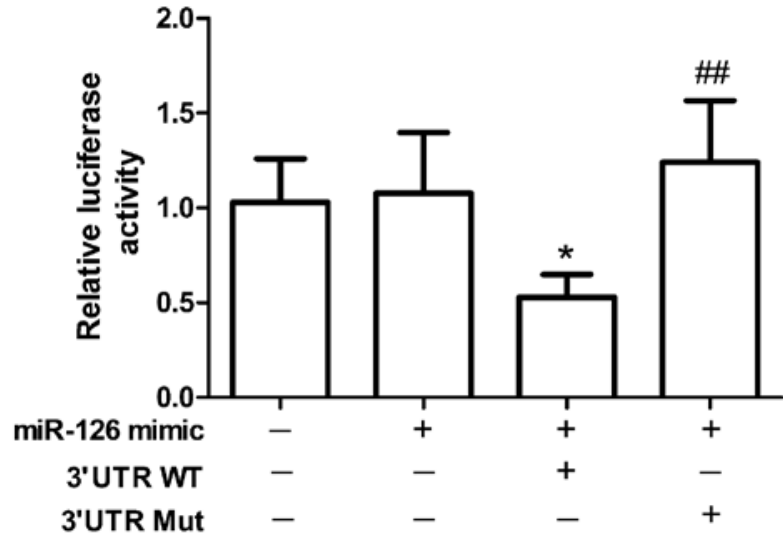

Figure 3. Vascular cell adhesion molecule-1 (VCAM-1) as a target gene of miR-126. The partially complementary miR-126-binding site of VCAM-1 3'-UTR (or a mutant binding site) was inserted downstream of a luciferase reporter on the pLUC-report plasmid, and co-transfected into vascular endothelial cells (VECs) with miR-126 mimic. Data are expressed as the means $\pm \mathrm{SD}$ of at least independently triplicate experiments. " $\mathrm{P}<0.05$ vs. miR126 mimic group; ${ }^{\# \#} \mathrm{P}<0.01$ vs. miR-126 mimic and wild-type of 3 'UTR group. by transfection with the miR-126 mimic. Transfection with the miR-126 inhibitor led to an increase in VCAM-1 expression. Taken together, our results suggested that miR-126 had a lower expression in the ox-LDL-stimulated VECs, and that miR-126 negatively regulated VCAM-1 expression.

Identification of VCAM-1 serving as a target gene of miR-126. To determine the predicted target sites for miR-126 in the 3'UTR of VCAM-1 mRNA which is responsible for the downregulation of VCAM-1 expression, dual-luciferase reporter assay with a vector containing the VCAM-1 wild-type or mutant-type 3'UTR downstream of the luciferase reporter gene was performed. The wild-type or mutant-type 3'UTR mRNA of VCAM-1 and miR-126 mimic were transfected into the VECs using HiPerFect reagent. Following $24 \mathrm{~h}$ of culture, firefly luciferase activity was detected in the cells transfected with the reporter plasmids and normalized to the transfection efficiency with Renilla luciferase activity, which was derived from a 
A

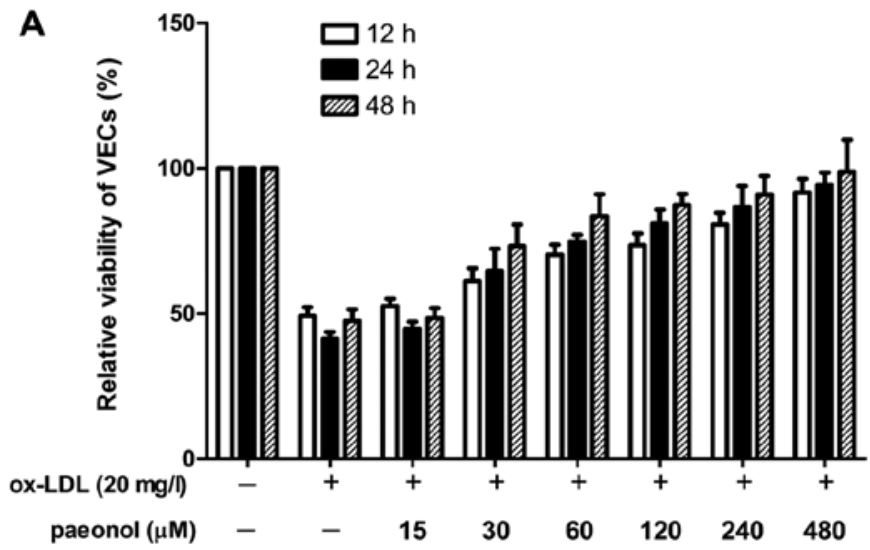

B

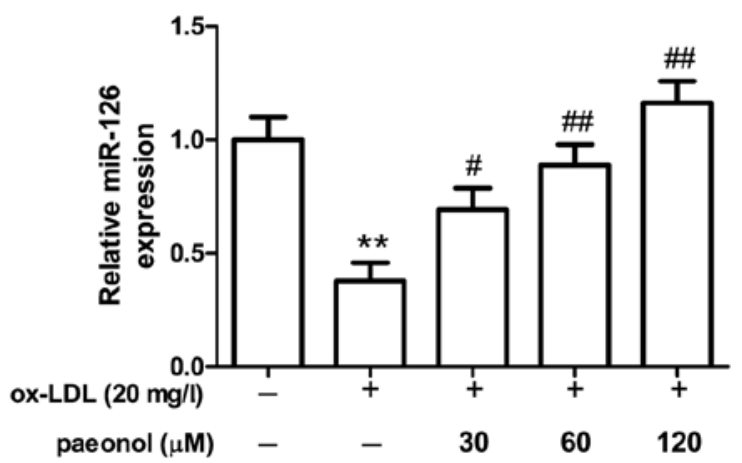

Figure 4. Effect of paeonol on miR-126 expression in oxidized low-density lipoprotein (ox-LDL)-injured vascular endothelial cells (VECs). (A) Effect of paeonol $(15,30,60,120,240$ and $480 \mu \mathrm{M})$ on ox-LDL-injured VECs at 12, 24 and $48 \mathrm{~h}$ as determined by MTT assay. (B) VECs were pre-treated with various concentrations of paeonol $(30,60$ and $120 \mu \mathrm{M})$, and miR-126 expression was examined by qPCR. Data are expressed as the means \pm SD of at least independently triplicate experiments. ${ }^{* *} \mathrm{P}<0.01$ vs. control group (no treatment); ${ }^{\#} \mathrm{P}<0.05$ and ${ }^{\# \#} \mathrm{P}<0.01$ vs. ox-LDL group.

A

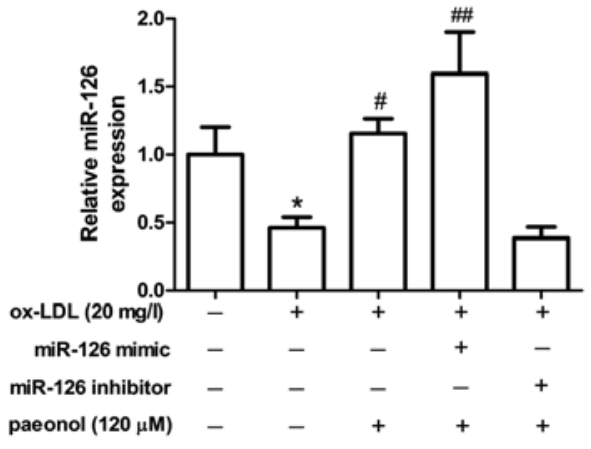

B

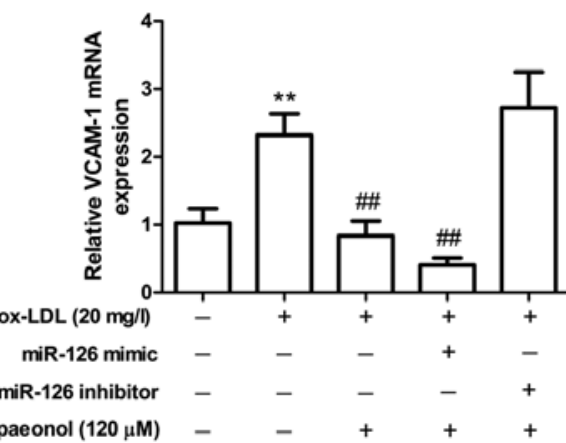

C
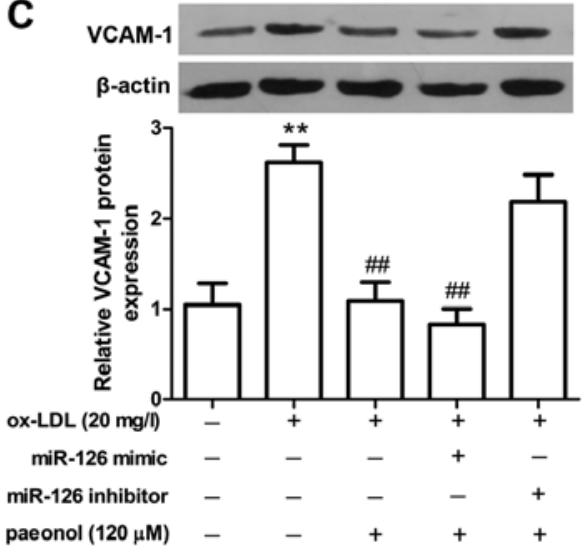

Figure 5.Effect of paeonol on vascular cell adhesion molecule-1 (VCAM-1) expression via miR-126 expression in oxidized low-density lipoprotein (ox-LDL)-injured vascular endothelial cells (VECs). (A) miR-126 mimic/inhibitor was transfected into rat VECs and after $24 \mathrm{~h}$, VECs were treated with paeonol (120 $\mu \mathrm{M}$ ) and then stimulated with ox-LDL ( $20 \mathrm{mg} / \mathrm{l})$ for $24 \mathrm{~h}$, and the relative miR-126 expression was analyzed by qPCR. (B) Relative mRNA expression levels of VCAM-1 in VECs were detected by qPCR. (C) The protein expression of VCAM-1 relative to $\beta$-actin was examined by western blot analysis. Data are expressed as the means \pm SD of at least independently triplicate experiments. ${ }^{*} \mathrm{P}<0.05$ and ${ }^{* *} \mathrm{P}<0.05$ vs. control group (no treatment); ${ }^{\#} \mathrm{P}<0.05$ and ${ }^{\# \#} \mathrm{P}<0.01$ vs. ox-LDL group.

co-transfection with an expression plasmid. Transfection with the wild-type plasmids and the presence of miR-126 target sites led to a marked reduction in luciferase activity. By contrast, the incorporation of mutant-type 3'UTR mRNA plasmids did not affect luciferase efficiency, although the luciferase activity was increased compared to the group transfected with the wildtype plasmid (Fig. 3). It was clearly demonstrated that VCAM-1 served as a target gene of miR-126 in the VECs.

Effect of paeonol on miR-126 expression in ox-LDL-injured $V E C s$. To further determine the mechanisms through which paeonol can effectively prevent and treat AS, we examined the effect of paeonol on miR-126 expression in the ox-LDL-injured VECs. The VECs were first treated with paeonol, and then stimulated with ox-LDL $(20 \mathrm{mg} / \mathrm{l})$. We found that treatment with paeonol $(30,60,120,240$ and $480 \mu \mathrm{M})$ protected the VECs from ox-LDL-induced injury, as shown by the increase in cell viability (Fig. 4A). However, paeonol had a slightly toxic effect as a concentration $>480 \mu \mathrm{M}$ and cultured times longer than 48 h (data not shown). Notably, paeonol significantly promoted miR-126 expression in a concentration-dependent manner (Fig. 4B). Our resutls thus suggested that paeonol promoted miR-126 expression to inhibit VEC injury.

Paeonol exerts inhibitory effects on VCAM-1 expression by promoting miR-126 expression. In order to demonstrate the effect of paeonol on the function of miR-126, we analyzed the expression of its target gene, VCAM-1. Firstly, the mimic/inhibitor of miR-126 was transfected into rat VECs; after $24 \mathrm{~h}$, the VECs were cultured with medium containing paeonol $(120 \mu \mathrm{M})$. We found that the miR-126 expression level was markedly upregulated by paeonol, increased by the miR-126 mimic, and decreased by the miR-126 inhibitor (Fig. 5A). Paeonol markedly suppressed the mRNA expression of VCAM-1, and the overexpression of miR-126 induced by transfection with the miR-126 mimic markedly inhibited the mRNA expression of VCAM-1; this effect of paeonol was attenuated by transfection with the miR-126 inhibitor (Fig. 5B). We then sought to determine the regulatory effect of miR-126 mediated by paeonol on VCAM-1 expression at the protein level. The upregulation of miR-126 


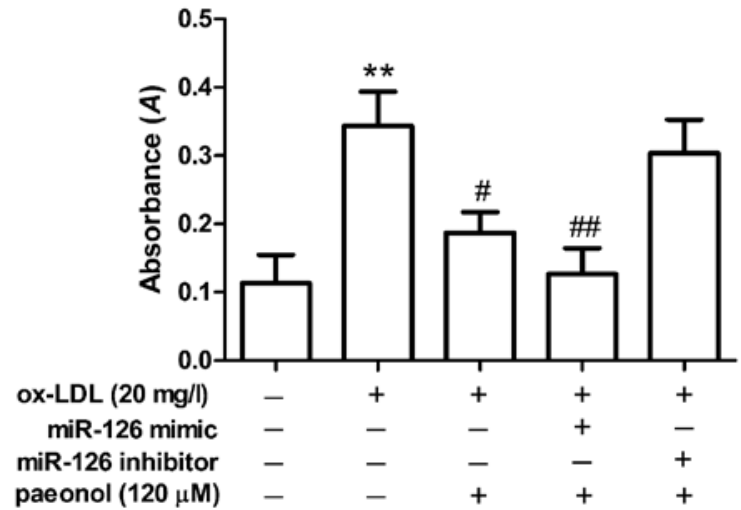

Figure 6. Effect of paeonol on monocyte adhesion to oxidized low-density lipoprotein (ox-LDL)-injured vascular endothelial cells (VECs) via miR-126 expression. Rose-Bengal dye was added to the pre-treated VECs, followed by incubation for $10 \mathrm{~min}$. The monocyte adhesion to VECs was detected using a microplate reader at $490 \mathrm{~nm}$. Data are expressed as the means $\pm \mathrm{SD}$ of at least independently triplicate experiments. ${ }^{* *} \mathrm{P}<0.05$ vs. control group (no treatment); ${ }^{\#} \mathrm{P}<0.05$ and ${ }^{\# \#} \mathrm{P}<0.01$ vs. ox-LDL group.

expression by transfection with miR-126 mimic markedly enhanced the inhibitory effect of paeonol on VCAM-1 protein expression. By contrast, the knockdown of miR-126 markedly diminished the inhibitory effects of paeonol on VCAM-1 expression (Fig. 5C). These results indicated that paeonol inhibited VCAM-1 expression by promoting miR-126 expression.

A
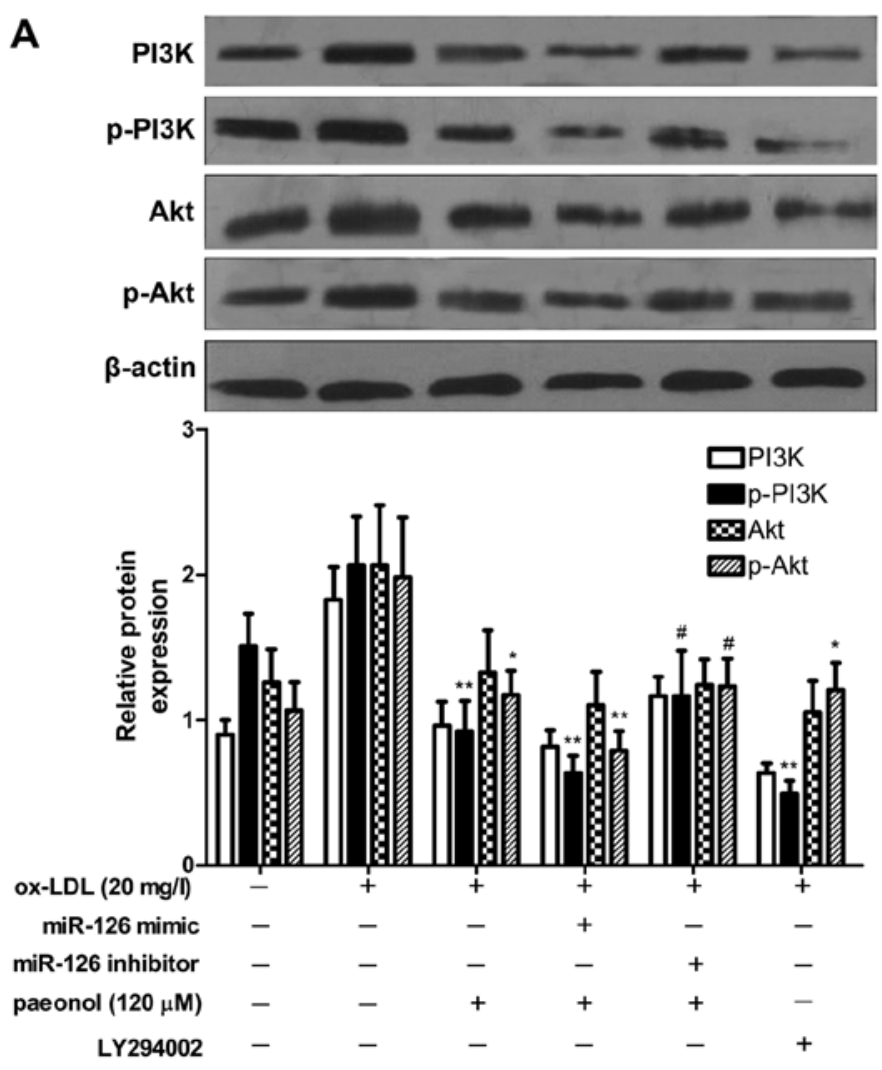

Effects of paeonol on monocyte adhesion to ox-LDL-injured $V E C s$ are mediated via miR-126 expression. To investigate whether paeonol inhibits monocyte adhesion to ox-LDL-injured VECs by regulating miR-126 expression, the mimic/inhibitor of miR-126 was transfected into the VECs; after $24 \mathrm{~h}$, the VECs were stimulated with ox-LDL in the presence or absence of paeonol. ox-LDL induced monocyte adhesion to VECs. It was shown that paeonol suppressed monocyte adhesion to VECs induced by ox-LDL, and the effect of paeonol was enhanced by the miR-126 mimic, but was reduced by the miR-126 inhibitor (Fig. 6). These results suggested that paeonol suppressed monocyte adhesion to ox-LDL-injured VECs via miR-126 expression.

Effects of paeonol on the PI3K/Akt/NF- $\kappa B$ pathway are mediated via miR-126 expression. To explore whether the promoting effects of paeonol on miR-126 expression are closely related to the PI3K/Akt/NF- $\mathrm{KB}$ pathway, miR-126 mimic/inhibitor was transfected into the VECs. After $24 \mathrm{~h}$, the VECs were treated with LY294002 (an inhibitor of PI3K) and PDTC (an inhibitor of NF- $\kappa B$ ) for $1 \mathrm{~h}$, and then stimulated with ox-LDL $(20 \mathrm{mg} / \mathrm{l})$ in the presence of paeonol $(120 \mu \mathrm{M})$. Paeonol significantly suppressed the phosphorylation of PI3K and Akt. The effect of paeonol was enhanced by transfection with the miR-126 mimic, but was diminished by transfection with the miR-126 inhibitor (Fig. 7A). Moreover, it was found that paeonol significantly suppressed the phosphorylation of

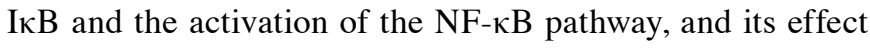

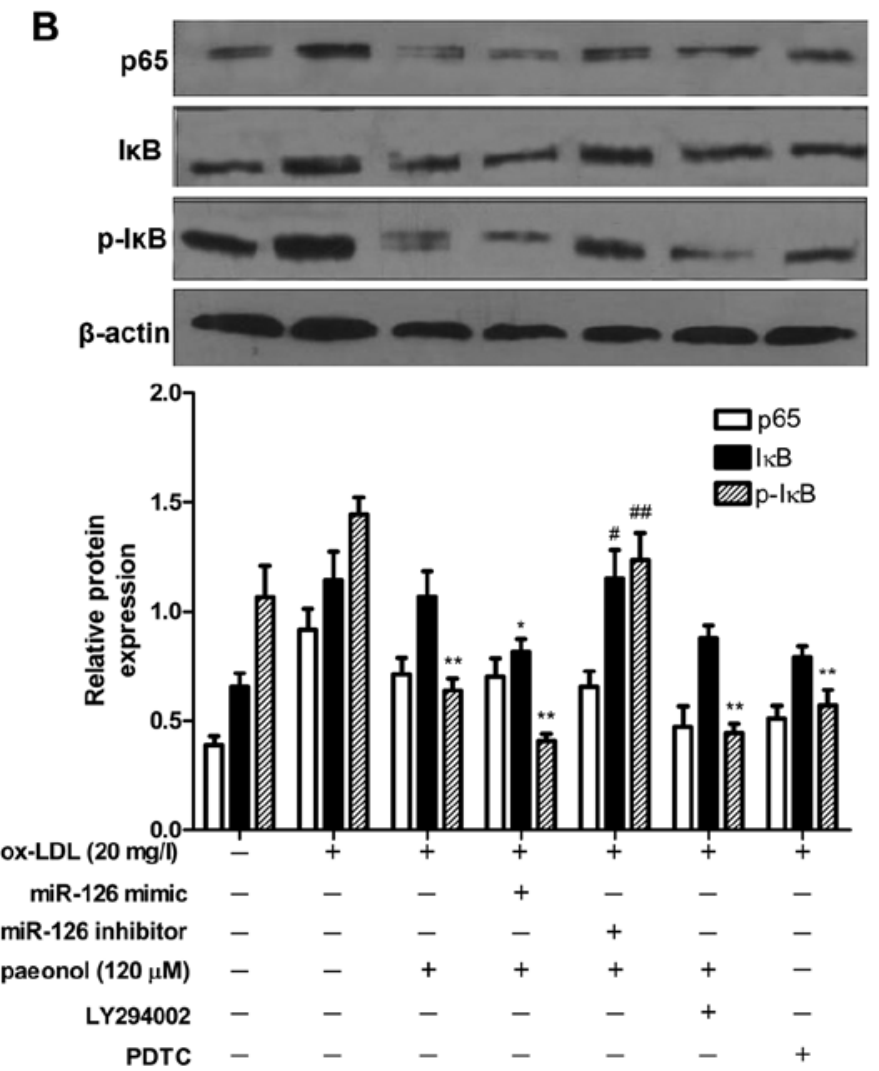

Figure 7. Effect of paeonol on phosphatidylinositol 3-kinase (PI3K)/protein kinase B (Akt)/nuclear factor- $\kappa \mathrm{B}(\mathrm{NF}-\kappa \mathrm{B})$ pathway via miR-126 expression. (A) Effect of paeonol on PI3K, p-PI3K, Akt and p-Akt protein expression was measured by western blot analysis, and normalized to $\beta$-actin expression. (B) Effect of

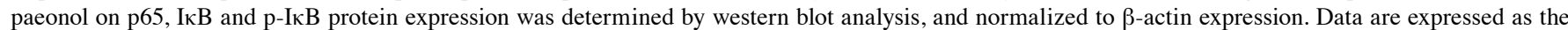
means \pm SD of at least independently triplicate experiments. ${ }^{*} \mathrm{P}<0.05$ and ${ }^{* *} \mathrm{P}<0.01$ vs. oxidized low-density lipoprotein (ox-LDL) group; ${ }^{\#} \mathrm{P}<0.05$ and ${ }^{\# \#} \mathrm{P}<0.01$ vs. ox-LDL, paeonol and miR-126 mimic treatment group. 
was enhanced by the miR-126 mimic, but diminished by the miR-126 inhibitor. In addition, the $\mathrm{NF}-\kappa \mathrm{B}$ pathway was indicated as downstream of the PI3K/Akt pathway by the inhibitor of the PI3K pathway (Fig. 7B). Taken together, our results revealed that paeonol blocked the activation of the $\mathrm{PI} 3 \mathrm{~K} / \mathrm{Akt} / \mathrm{NF}-\kappa \mathrm{B}$ signaling pathway via the upregulation of miR-126 expression.

\section{Discussion}

In this study, ox-LDL markedly induced damage to VECs, and miR-126 had a lower expression in ox-LDL-injured VECs in vitro. It has been reported that miR-126 is a strongly expressed miRNA specific to endothelial cells, and also affects the physiological and pathological processes of vascular diseases, such as AS $(11,21)$. AS is a complex chronic inflammatory and metabolic disease (22). Injured VECs may be detrimental to the structure of plaque, and based on the fact that endothelial destruction precedes atherogenesis, the injury of VECs may also predispose to arterial thrombosis (23). VCAM-1 plays an important role in the slow rolling of monocytes along the surface of the endothelial layer and in the pathological process of AS $(9,24)$. miRNAs play an important role in both inflammatory and cardiovascular diseases (25). In this study, miR-126 downregulated VCAM-1 expression and protected the rat VECs from ox-LDL-induced injury.

miRNAs can bind and interfere with the mRNAs of target genes specifically, and negatively regulate their expression. Accordingly, in this study, miR-126 suppressed VCAM-1 expression by combination of the miR-126-binding sites, and the upregulation of miR-126 inhibited VCAM-1 gene expression. Exogenous miR-126 suppresses luciferase activity when the miR-126-binding site is fused to the luciferase 3'UTR mRNA of VCAM-1 (11). These findings raise the possibility that medical strategies aimed at causing controlled alterations to the levels of gene-targeting miRNAs, such as miR-126, may be effective therapies for inflammatory diseases. miRNAs are good candidates, particularly for the therapeutic intervention, as they have an impact on the expression of target genes, rather than simply turning genes on or off, thereby regulating cellular inflammation through external regulation.

Moreover, we found that paeonol promoted miR-126 expression to suppress VCAM-1 expression in ox-LDL-injured VECs and monocyte adhesion to the VECs. miR-126 expression can be modulated by natural compound, and participates in the vascular inflammatory response (26). The deletion of miR-126 causes the loss of vascular integrity and leads to defects in the proliferation and migration of endothelial cells (27). VCAM-1 is expressed in VECs and plays an important role in monocyte or lymphocyte rolling and adhesion in inflammation in earlyonset AS $(28,29)$. Thus, the inhibition of VCAM-1 expression suggests anti-inflammatory properties for endothelial cells As reported in a previous study, paeonol suppressed VCAM-1 expression in TNF- $\alpha$-stimulated VECs (3). VCAM-1 expression can be regulated at the post-transcriptional level (30). miR-126 inhibits leukocyte adherence through the decrease of VCAM-1 expression (11). In is suggested that miR-126 expression induced by paeonol can downregulate the expression of adhesion molecule and may exert additional inhibitory effects on vascular inflammation.
However, it has been demonstrated that the transcription factor, $\mathrm{NF}-\kappa \mathrm{B}$, is sensitve to oxidants such as ox-LDL, and is also associated with the cellular function of adhesion $(31,32)$. $\mathrm{NF}-\kappa \mathrm{B}$ activation is required for the induction of adhesion molecules, such as VCAM-1, and is responsible for monocyte adhesion and vascular inflammation (33). On the other hand, the $\mathrm{PI} 3 \mathrm{~K} / \mathrm{Akt}$ signaling pathway is known as a critical regulator of $\mathrm{NF}-\kappa \mathrm{B}$ activation in response to extracellular stress (34). In this study, to further clarify the detailed mechanisms responsible for the inhibitory effects of paeonol on cell adhesion, the effects of paeonol on the PI3K/Akt/NF- $\kappa \mathrm{B}$ pathway were analyzed. Our results revealed that paeonol blocked the activation of the $\mathrm{PI} 3 \mathrm{~K} / \mathrm{Akt} / \mathrm{NF}-\kappa \mathrm{B}$ signaling pathway by promoting miR-126 expression. The overexpression of miR-126 has been reported to inhibit the expression of $\mathrm{p}$-Akt and to regulate PI3K signaling by targeting the PI3K regulatory subunit $\beta(\mathrm{p} 85 \beta)(35,36)$ a target gene of miR-126 identified previously in endothelial cells (37), indicating that its function is related to the PI3K/Akt pathway. Similarly, the decrease in p-Akt protein levels follwoing the suppression of $\mathrm{p} 85 \beta$ protein expression by $\mathrm{miR}-126$ has also reported (38). In this study, it was proven that $\mathrm{NF}-\kappa \mathrm{B}$ is downstream of the PI3K/Akt pathway as regards the effect of paeonol. It played an important role in the inhibitory effect of paeonol on monocyte adhesion to ox-LDL-injured VECs via the induction of miR-126 expression.

In conclusion, in the present study, we demonstrated that paeonol promoted miR-126 expression to inhibit monocyte adhesion to ox-LDL-injured VECs and block the activation of the PI3K/Akt/NF- $\mathrm{B}$ signaling pathway. We provide evidence that miR-126 may serve as an important therapeutic target in the treatment of AS with the use of paeonol.

\section{Acknowledgements}

This study was supported by research grants from the National Natural Science Foundation of China (grant. nos. 81473386 and 81274134).

\section{References}

1. Chou TC: Anti-inflammatory and analgesic effects of paeonol in carrageenan-evoked thermal hyperalgesia. Br J Pharmacol 139: 1146-1152, 2003.

2. Nizamutdinova IT, Jin YC, Kim JS, Yean MH, Kang SS, Kim YS Lee JH, Seo HG, Kim HJ and Chang KC: Paeonol and paeoniflorin, the main active principles of Paeonia albiflora, protect the heart from myocardial ischemia/reperfusion injury in rats. Planta Med 74: 14-18, 2008.

3. Pan LL and Dai M: Paeonol from Paeonia suffruticosa prevents TNF-alpha-induced monocytic cell adhesion to rat aortic endothelial cells by suppression of VCAM-1 expression. Phytomedicine 16: 1027-1032, 2009.

4. Hsieh CL, Cheng CY, Tsai TH, Lin IH, Liu CH, Chiang SY, Lin JG, Lao CJ and Tang NY: Paeonol reduced cerebral infarction involving the superoxide anion and microglia activation in ischemia-reperfusion injured rats. J Ethnopharmacol 106: 208-215, 2006.

5. Su SY, Cheng CY, Tsai TH, Hsiang CY, Ho TY and Hsieh CL: Paeonol attenuates $\mathrm{H}_{2} \mathrm{O}_{2}$-induced NF- $\mathrm{KB}$-associated amyloid precursor protein expression. Am J Chin Med 38: 1171-1192, 2010.

6. Vitaglione P, Morisco F, Caporaso N and Fogliano V: Dietary antioxidant compounds and liver health. Crit Rev Food Sci Nutr 44: 575-586, 2004.

7. Li H, Dai M and Jia W: Paeonol attenuates high-fat-diet-induced atherosclerosis in rabbits by anti-inflammatory activity. Planta Med 75: 7-11, 2009. 
8. Li W, Ishihara K, Yokota T, Nakagawa T, Koyama N, Jin J, Mizuno-Horikawa Y, Wang X, Miyoshi E, Taniguchi N and Kondo A: Reduced alpha4betal integrin/VCAM-1 interactions lead to impaired pre-B cell repopulation in alpha 1,6-fucosyltransferase deficient mice. Glycobiology 18: 114-124, 2008

9. Hwa JS, Mun L, Kim HJ, Seo HG, Lee JH, Kwak JH, Lee DU and Chang KC: Genipin selectively inhibits TNF- $\alpha$-activated VCAM-1 but not ICAM-1 expression by upregulation of PPAR- $\gamma$ in endothelial cells. Korean J Physiol Pharmacol 15: 157-162, 2011.

10. Tsoyi K, Kim WS, Kim YM, Kim HJ, Seo HG, Lee JH, Yun-Choi HS and Chang KC: Upregulation of PTEN by CKD712, a synthetic tetrahydroisoquinoline alkaloid, selectively inhibits lipopolysaccharide-induced VCAM-1 but not ICAM-1 expression in human endothelial cells. Atherosclerosis 207: 412-419, 2009.

11. Harris TA, Yamakuchi M, Ferlito M, Mendell JT and Lowenstein CJ: MicroRNA-126 regulates endothelial expression of vascular cell adhesion molecule 1. Proc Natl Acad Sci USA 105: 1516-1521, 2008.

12. Sturgeon CM, Chicha L, Ditadi A, Zhou Q, McGrath KE, Palis J, Hammond SM, Wang S, Olson EN and Keller G: Primitive erythropoiesis is regulated by miR-126 via nonhematopoietic VCAM-1 ${ }^{+}$cells. Dev Cell 23: 45-57, 2012.

13. Harris TA, Yamakuchi M, Kondo M, Oettgen P and Lowenstein CJ: Ets-1 and Ets-2 regulate the expression of microRNA-126 in endothelial cells. Arterioscler Thromb Vasc Biol 30: 1990-1997, 2010.

14. Nikolic I, Plate KH and Schmidt MH: EGFL7 meets miRNA-126: An angiogenesis alliance. J Angiogenes Res 2: 9, 2010.

15. Bai Y, Bai X, Wang Z, Zhang X, Ruan C and Miao J: MicroRNA-126 inhibits ischemia-induced retinal neovascularization via regulating angiogenic growth factors. Exp Mol Pathol 91: 471-477, 2011.

16. Sui L, Wang J and Li BM: Role of the phosphoinositide 3-kinaseAkt-mammalian target of the rapamycin signaling pathway in long-term potentiation and trace fear conditioning memory in rat medial prefrontal cortex. Learn Mem 15: 762-776, 2008.

17. Tsubosaka Y, Murata T, Yamada K, Uemura D, Hori M and Ozaki H: Halichlorine reduces monocyte adhesion to endothelium through the suppression of nuclear factor-kappaB activation. J Pharmacol Sci 113: 208-213, 2010

18. Ghosh S, Banerjee S and Sil PC: The beneficial role of curcumin on inflammation, diabetes and neurodegenerative disease: A recent update. Food Chem Toxicol 83: 111-124, 2015.

19. Salvayre R, Auge N, Benoist H and Negre-Salvayre A: Oxidized low-density lipoprotein-induced apoptosis. Biochim Biophys Acta 1585: 213-221, 2002.

20. Basoni C, Nobles M, Grimshaw A, Desgranges C, Davies D, Perretti M, Kramer IM and Genot E: Inhibitory control of TGF-beta1 on the activation of Rap1, CD11b, and transendothelial migration of leukocytes. FASEB J 19: 822-824, 2005.

21. Asgeirsdóttir SA, van Solingen C, Kurniati NF, Zwiers PJ, Heeringa P, van Meurs M,Satchell SC, Saleem MA, Mathieson PW, Banas B, et al: MicroRNA-126 contributes to renal microvascular heterogeneity of VCAM-1 protein expression in acute inflammation. Am J Physiol Renal Physiol 302: F1630-F1639, 2012.

22. Rioufol G, Finet G, Ginon I, André-Fouët X, Rossi R, Vialle E, Desjoyaux E, Convert G, Huret JF and Tabib A: Multiple atherosclerotic plaque rupture in acute coronary syndrome: A three-vessel intravascular ultrasound study. Circulation 106: 804-808, 2002.

23. Peng N, Meng N, Wang S, Zhao F, Zhao J, Su L, Zhang S, Zhang Y, Zhao B and Miao J: An activator of mTOR inhibits oxLDL-induced autophagy and apoptosis in vascular endothelial cells and restricts atherosclerosis in apolipoprotein $\mathrm{E}^{-/-}$mice. Sci Rep 4: 5519, 2014
24. Fuentes E, Palomo I and Alarcón M: Platelet miRNAs and cardiovascular diseases. Life Sci 133: 29-44, 2015.

25. Condorelli G, Latronico MV and Cavarretta E: microRNAs in cardiovascular diseases: Current knowledge and the road ahead. J Am Coll Cardiol 63: 2177-2187, 2014.

26. Angel-Morales G, Noratto G and Mertens-Talcott SU: Standardized curcuminoid extract (Curcuma longa L.) decreases gene expression related to inflammation and interacts with associated microRNAs in human umbilical vein endothelial cells (HUVEC). Food Funct 3: 1286-1293, 2012.

27. Wang S, Aurora AB, Johnson BA, Qi X, McAnally J, Hill JA, Richardson JA, Bassel-Duby R and Olson EN: The endothelialspecific microRNA miR-126 governs vascular integrity and angiogenesis. Dev Cell 15: 261-271, 2008

28. Cybulsky MI, Iiyama K, Li H, Zhu S, Chen M, Iiyama M, Davis V, Gutierrez-Ramos JC, Connelly PW and Milstone DS: A major role for VCAM-1, but not ICAM-1, in early atherosclerosis. J Clin Invest 107: 1255-1262, 2001.

29. Libby P: Inflammation in atherosclerosis. Nature 420: $868-874$, 2002.

30. Croft D, McIntyre P, Wibulswas A and Kramer I: Sustained elevated levels of VCAM-1 in cultured fibroblast-like synoviocytes can be achieved by TNF-alpha in combination with either IL-4 or IL-13 through increased mRNA stability. Am J Pathol 154: 1149$1158,1999$.

31. Hayden MS and Ghosh S: Signaling to NF-kappaB. Genes Dev 18: 2195-2224, 2004.

32. Albelda SM, Smith CW and Ward PA: Adhesion molecules and inflammatory injury. FASEB J 8: 504-512, 1994.

33. Lockyer JM, Colladay JS, Alperin-Lea WL, Hammond T and Buda AJ: Inhibition of nuclear factor-kappaB-mediated adhesion molecule expression in human endothelial cells. Circ Res 82: 314-320, 1998.

34. Yang M, Huang J, Pan HZ and Jin J: Triptolide overcomes dexamethasone resistance and enhanced PS-341-induced apoptosis via PI3k/Akt/NF- $\mathrm{kB}$ pathways in human multiple myeloma cells. Int J Mol Med 22: 489-496, 2008.

35. Wang XC, Du LQ, Tian LL, Wu HL, Jiang XY, Zhang H, Li DG, Wang YY, Wu HY, She Y, et al: Expression and function of miRNA in postoperative radiotherapy sensitive and resistant patients of non-small cell lung cancer. Lung Cancer 72: 92-99, 2011.

36. Guo C, Sah JF, Beard L, Willson JK, Markowitz SD and Guda K: The noncoding RNA, miR-126, suppresses the growth of neoplastic cells by targeting phosphatidylinositol 3-kinase signaling and is frequently lost in colon cancers. Genes Chromosomes Cancer 47: 939-946, 2008.

37. Fish JE, Santoro MM, Morton SU, Yu S, Yeh RF, Wythe JD, Ivey KN, Bruneau BG, Stainier DY and Srivastava D: miR-126 regulates angiogenic signaling and vascular integrity. Dev Cell 15: 272-284, 2008 .

38. Sessa R, Seano G, di Blasio L, Gagliardi PA, Isella C, Medico E, Cotelli F, Bussolino F and Primo L: The miR-126 regulates angiopoietin-1 signaling and vessel maturation by targeting p85ß. Biochim Biophys Acta 1823: 1925-1935, 2012. 